\title{
Deformations of circle-valued functions on 2-torus
}

\author{
Bohdan Feshchenko
}

\begin{abstract}
In this paper we give an algebraic description of fundamental groups of orbits of circle-valued smooth functions from some subspace of the space of smooth functions with isolated singularities on 2-torus $T^{2}$ with respect to the action of the group of diffeomorphisms of $T^{2}$.

Анотація. Нехай $M$ - гладка компактна поверхня. Через $P$ будемо позначати або $\mathbb{R}$ або $S^{1}$. Група дифеоморфізмів $\mathcal{D}(M)$ діє на просторі гладких відображень $C^{\infty}(M, P)$ за таким правилом: $\gamma: C^{\infty}(M, P) \times \mathcal{D}(M) \rightarrow$ $C^{\infty}(M, P), \gamma(f, h)=f \circ h$. Нехай $\mathcal{O}(f)=\{f \circ h \mid h \in \mathcal{D}(M)\}$ - орбіта $f$ відносно дії $\gamma$ і $\mathcal{O}_{f}(f)$ - зв'язна компонента $\mathcal{O}(f)$, що містить $f$. У статті досліджується алгебраїчна структура фундаментальної групи $\mathcal{O}_{f}(f)$ для широкого класу гладких функцій у коло на 2-торі, що мають ізольовані критичні точки.
\end{abstract}

\section{INTRODUCTION}

Morse functions on manifolds are one of the main objects in mathematics nowadays. It is well known that analytic properties of such functions carry information about the geometry and topology of the manifold on which they are defined [32].

Deformational properties of Morse functions were studied by many authors. For example, homotopy properties of connected components of spaces of Morse functions on smooth surfaces were studied by V. Sharko [40], H. Zieschang, S. Matveev, E. Kudryavtseva [17]. Cobordism groups of Morse functions on surfaces were calculated by K. Ikegami, O. Saeki [14] and B. Kalmar [15].

The paper is devoted to the study of circle-valued smooth functions from some subspace of smooth functions with isolated singularities on smooth

Keywords: Circle-valued functions, orbits, stabilizers, fundamental group

Ключові слова: відображення в коло, орбіти, стабілізатори, фундаментальна група DOI: http://dx.doi.org/10.15673/tmgc.v14i2.2008 
compact oriented surfaces and homotopy properties of special subspaces of such functions called orbits. The main example of such functions is circle-valued Morse functions which are natural generalizations of (ordinary) Morse functions. Circle-valued Morse functions can be viewed locally as functions but global properties of such functions are different from real-valued case. A modern theory of circle-valued Morse functions originates in a series of papers [33,34] by S. Novikov in 80 's. It was motivated by the study of multi-valued Lagrangians in some problems of theoretical physics and leaded him to develop a generalization of a Morse theory for circle-valued Morse functions and more generally a theory (now called Morse-Novikov theory) for differential 1-forms. This theory has many applications, e.g., in questions of fibrations of manifolds over $S^{1}$ [37], Lagrangian intersections [8], knot theory [41], Seiberg-Witten theory [12,13], etc. The reader can find more on this theory and its applications in the book by A. Pajitnov [35].

Recall that there is a natural action of the group of diffeomorphisms $\mathcal{D}(M)$ of a smooth compact surface $M$ on the space of smooth $P$-valued functions for $P=\mathbb{R}$ or $S^{1}$ given by the rule:

$$
\gamma: C^{\infty}(M, P) \times \mathcal{D}(M) \rightarrow C^{\infty}(M, P), \quad \gamma(f, h)=f \circ h .
$$

We consider stabilizers $\mathcal{S}(f)$ and orbits $\mathcal{O}(f)$ of a smooth function $f \in$ $C^{\infty}(M, P)$ with respect to the action $\gamma$ and their connected components $\mathcal{S}_{\text {id }}(f)$ and $\mathcal{O}_{f}(f)$ of the identity map $\operatorname{id}_{M}$ and a connected component containing $f$ respectively in the topologies induced from strong Whitney topologies on $\mathcal{D}(M)$ and $C^{\infty}(M, P)$ (see definitions in subsection 2.1).

We restrict out attention to the class of smooth $P$-valued functions $\mathcal{F}$ on a smooth compact surface which satisfies two conditions: functions take constant values on each boundary component and near every critical point can be represented as homogeneous polynomial $\mathbb{R}^{2} \rightarrow \mathbb{R}$ of degree $\geqslant 2$ without multiple factors (for definition of this class of functions $\mathcal{F}$, see subsection 2.2). It is well-known that the class $\mathcal{F}$ consists of "generic" functions with "topologically generic" singularities ([26, Section 3,4], subsection 2.2). Thus our restriction is insignificant, since the class $\mathcal{F}$ is "wide" enough.

S. Maksymenko $[20,22,23,25,27]$ showed that if $f: M \rightarrow P$ is a smooth function from $\mathcal{F}$ and $f$ has at least one saddle point, then $\pi_{n} \mathcal{O}_{f}(f)=\pi_{n} M$ for $n \geqslant 3, \pi_{2} \mathcal{O}_{f}(f)=0$ and for $\pi_{1} \mathcal{O}_{f}(f)$ there is a short exact ${ }^{1}$ sequence of groups

$$
\pi_{1} \mathcal{D}_{\mathrm{id}}(M) \stackrel{\zeta_{1}}{\longrightarrow} \pi_{1} \mathcal{O}_{f}(f) \stackrel{\partial_{1}}{\longrightarrow} \pi_{0} \mathcal{S}^{\prime}(f),
$$

\footnotetext{
${ }^{1}$ Throughout the text $\hookrightarrow$ and $\rightarrow$ mean mono- and epimorphism respectively.
} 
where $\mathcal{S}^{\prime}(f)$ is the group of $f$-preserving diffeomorphisms of $M$ which are isotopic to the identity map, see Eq. (2.1). This exact sequence is a nontrivial part of long exact sequence of homotopy groups of some fibration $\zeta_{f}$ : $\mathcal{D}_{\text {id }}(M) \rightarrow \mathcal{O}_{f}(f)$ (Theorem 2.4.1). As the consequence if $M$ is aspherical surface then all homotopy information is contained in $\pi_{1} \mathcal{O}_{f}(f)$, and the orbit $\mathcal{O}_{f}(f)$ itself is an Eilenberg-MacLane space $K\left(\pi_{1} \mathcal{O}_{f}(f), 1\right)$. So there are two natural questions about homotopy properties of $\mathcal{O}_{f}(f)$ : description of an algebraic structure of groups $\pi_{1} \mathcal{O}_{f}(f)$ and homotopy type of $\mathcal{O}_{f}(f)$.

An algebraic structure of $\pi_{1} \mathcal{O}_{f}(f)$ "partially" depends on homotopy properties of $\mathcal{D}_{\text {id }}(M)$ which were studied in $[3-5,10]$. It is well-known that if $M$ is a closed compact and oriented surface of genus $\geqslant 2$, then $\mathcal{D}_{\text {id }}(M)$ is contractible $[3-5,10]$, and so an epimorphism $\partial_{1}$ from $(2.3)$ is an isomorphism. Thus in this case the question on an algebraic structure of $\pi_{1} \mathcal{O}_{f}(f)$ reduces to the study of an algebraic structure of groups $\pi_{0} \mathcal{S}^{\prime}(f)$, which are easier to compute.

It is also known that the group $\mathcal{D}_{\text {id }}\left(T^{2}\right)$ for 2-torus is not contractible, so the image of $\pi_{1} \mathcal{D}_{\text {id }}\left(T^{2}\right) \cong \mathbb{Z}^{2}$ is non-trivial in $\pi_{1} \mathcal{O}_{f}(f)$. The sequence $(2.3)$ for functions on $T^{2}$ in general does not split [28]. So for this case we need an additional study.

An algebraic structure of $\pi_{1} \mathcal{O}_{f}(f)$ for real-valued functions from the class $\mathcal{F}$ on 2 -torus $T^{2}$ was studied in the series of papers by S. Maksymenko and the author [7,28-30]. Sufficient conditions for splitting the sequence (2.3) were obtained in [28]. It was shown that the question on an algebraic structure of $\pi_{1} \mathcal{O}_{f}(f)$ is reduced to studying algebraic structure of orbits and stabilizers for restrictions of a given function to subsurfaces of $T^{2}$ being 2disks and cylinders, for which this structure is known [27]. These cylinders and 2-disks are in some sense "building blocks" which carry "combinatorial symmetries" of the function.

The group $\pi_{0} \mathcal{S}^{\prime}(f)$ contains a lot of information about an algebraic structure of $\pi_{1} \mathcal{O}_{f}(f)$ and the homotopy type of $\mathcal{O}_{f}(f)$. Note that each diffeomorphism from $\mathcal{S}^{\prime}(f)$ induces an automorphism of Kronrod-Reeb graph $\Gamma_{f}$ of $f$. The group $\pi_{0} \mathcal{S}^{\prime}(f)$ contains a free abelian subgroup generated by Dehn twists which induce trivial action on $\Gamma_{f}$ and the corresponding quotient group $G(f)$ contains "discrete combinatorial" symmetries of the function $f$. So the group $\pi_{0} \mathcal{S}^{\prime}(f)$ can be viewed as "homeotopy" group for $f$-preserving and isotopic to the identity diffeomorphisms with $G(f)$ as its non-trivial counterpart.

It is known that the group $G(f)$ "controls" the homotopy type of $\mathcal{O}_{f}(f)$. In particular, if $f$ is generic, then the group $G(f)$ is trivial, and $\mathcal{O}_{f}(f)$ is homotopy equivalent to an $m$-torus $T^{m}$ if $M \neq S^{2}, M \neq \mathbb{R} P^{2}$, to $S^{2}$ if $M=S^{2}$ and $f$ has only two critical points, and to $\mathrm{SO}(3) \times T^{m}$ otherwise, 
for some $m \geqslant 0$ depending on $f$. E. Kudryavtseva $[18,19]$ calculated the homotopy types of connected components of the space of Morse functions on compact surfaces and generalized the result on homotopy types of orbits $\mathcal{O}_{f}(f)$ when the group $G(f)$ is non-trivial.

It is also known that if $f: M \rightarrow P$ has exactly $n$ critical points, then $\mathcal{O}_{f}(f)$ is homotopy equivalent to some covering space of $n^{\text {th }}$ configuration space of $M$ and $\pi_{1} \mathcal{O}_{f}(f)$ is a subgroup of $n^{\text {th }}$ braid group $B_{n}(M)$ [21, Theorem 2, Corollary 4]. A good overview the reader can find in [26, 27] where these results are presented in the form of so-called crystallographic and Bieberbach sequences.

Our main goal is to generalize our results on algebraic structure of $\pi_{1} \mathcal{O}_{f}(f)$ to the case of circle-valued functions from the class $\mathcal{F}$ on $T^{2}$.

Notice that if $f: T^{2} \rightarrow S^{1}$ is a smooth function without critical points, then $f: T^{2} \rightarrow S^{1}$ is a locally trivial fibration and the homotopy types of orbits and stabilizers are known, see [20, Theorem 1.9], so we will always assume that all functions have at least one critical point.

General overview of results. First we will show (see Theorem 3.1.1) that if $f: M \rightarrow S^{1}$ is a null-homotopic function from $\mathcal{F}$ then $\mathcal{O}_{f}(f)$ is homeomorphic to $\mathcal{O}_{\tilde{f}}(\tilde{f})$, where $\tilde{f}: M \rightarrow \mathbb{R}$ is also a smooth function from $\mathcal{F}$ which is a lift of $f$ with respect to the universal cover $p: \mathbb{R} \rightarrow S^{1}$. So for null-homotopic functions the problem in hand is completely reduced to the real-valued case, which is well studied.

Further we prove that for circle-valued functions with isolated singularities on 2-torus their Kronrod-Reeb graphs are either trees or contain a unique cycle, and if $f$ is not null-homotopic, then those graphs are not trees (Lemma 3.3.1).

In our main result (Theorem 4.1) we use an algebraic construction wreath product (subsection 3.4) - to describe an algebraic structure of $\pi_{1} \mathcal{O}_{f}(f)$ for functions from $\mathcal{F}$ on $T^{2}$ whose graphs contain a cycle via groups of connected components of stabilizers of the restrictions of a function $f$ onto cylinders.

It is well-known that $\pi_{1} \mathcal{D}_{\text {id }}\left(T^{2}\right)$ is free abelian group of rank $2([4,10])$; we define these two generators $\mathbf{L}$ and $\mathbf{M}$ adapted to some coordinate system on $T^{2}$ (see Eq. (5.1)). The image of $\mathbf{L}$ in $\pi_{1} \mathcal{O}_{f}(f)$ plays an important role in the proof of Theorem 4.1, but the image of $\mathbf{M}$ is "invisible" in our description of $\pi_{1} \mathcal{O}_{f}(f)$. Proposition 7.1 contains the information about image of $\mathbf{M}$ in $\pi_{1} \mathcal{O}_{f}(f)$.

The obtained results will be used to further study the orbits of smooth functions as well as their relationships with braid groups on surfaces mentioned above or more generally with Artin groups. 
Structure of the paper. In Section 2 we give definitions of stabilizers and orbits of smooth functions with values in $P$ on surfaces (subsection 2.1) and discuss their homotopy properties (Theorem 2.4.1) for functions from the class $\mathcal{F}$.

Section 3 contains auxiliary constructions such as null-homotopic functions to $S^{1}$ and their orbits (subsection 3.1), topological properties of graphs of circle-valued functions with isolated singularities on $T^{2}$ (subsection 3.2) and wreath product of special type (subsection 3.4). They will be needed to state our main result - Theorem 4.1 in Section 4.

Section 5 contains some additional constructions and result needed to the proof of Theorem 4.1 in Section 6 .

Finally, we study the "place" of the generator $\mathbf{M}$ of $\pi_{1} \mathcal{D}_{\text {id }}\left(T^{2}\right)$ in $\pi_{1} \mathcal{O}_{f}(f)$ (Section 7).

\section{Definitions AND useful FACts}

2.1. Orbits and stabilizers of smooth functions. Let $M$ be a smooth compact surface, $X$ be a closed (possible empty) subset of $M$, and $P$ denotes either $\mathbb{R}$ or $S^{1}$. The group $\mathcal{D}(M, X)$ of diffeomorphisms of $M$ fixed on $X$ acts from the right on the space of smooth maps $C^{\infty}(M, P)$ by the rule

$$
\gamma: C^{\infty}(M, P) \times \mathcal{D}(M, X) \rightarrow C^{\infty}(M, P), \quad \gamma(f, h)=f \circ h .
$$

With respect to $\gamma$ we denote by

$$
\begin{aligned}
& \mathcal{S}(f, X)=\{h \in \mathcal{D}(M, X) \mid f \circ h=f\}, \\
& \mathcal{O}(f, X)=\{f \circ h \mid h \in \mathcal{D}(M, X)\}
\end{aligned}
$$

the stabilizer and the orbit of $f \in C^{\infty}(M, P)$. Endow strong Whitney $C^{\infty}$ topologies on $C^{\infty}(M, P)$ and $\mathcal{D}(M, X)$; then for a map $f \in C^{\infty}(M, P)$ these topologies induce some topologies on $\mathcal{S}(f, X)$ and $\mathcal{O}(f, X)$. Denote by $\mathcal{D}_{\text {id }}(M, X), \mathcal{S}_{\text {id }}(f, X)$ the connected components of the identity map $\mathcal{D}(M, X)$ and $\mathcal{S}(f, X)$ respectively, and by $\mathcal{O}_{f}(f, X)$ the connected component of $\mathcal{O}(f, X)$ containing $f$. If $X=\varnothing$ we omit the symbol " $\varnothing$ " from our notation, i.e. write $\mathcal{D}(M)$ and $\mathcal{S}(f)$ instead of $\mathcal{D}(M, \varnothing)$ and $\mathcal{S}(f, \varnothing)$ and so on. We also set

$$
\mathcal{S}^{\prime}(f, X)=\mathcal{S}(f, X) \cap \mathcal{D}_{\text {id }}(M, X) .
$$

The group $\mathcal{S}^{\prime}(f, X)$ consists of isotopic to the identity $f$-preserving diffeomorphisms fixed on $X$.

2.2. The space $\mathcal{F}(M, P)$. Let $\mathcal{F}(M, P)$ be a subset of $C^{\infty}(M, P)$ satisfying the following conditions:

(1) the map $f$ takes constant values at each boundary component of $M$, 
(2) for every critical point $z$ of $f$ there are local coordinates in which $f$ is a homogeneous polynomial $\mathbb{R}^{2} \rightarrow \mathbb{R}$ of degree $\geqslant 2$ without multiple factors.

Notice that the class $\mathcal{F}$ is "natural" and "generic" class of smooth functions. It follows from 2 that every $f \in \mathcal{F}(M, P)$ has only isolated critical points, and thus the set of critical points of $f$ is finite. For instance the space of $P$-valued Morse functions, i.e. smooth maps $f: M \rightarrow P$ having only nondegenerate critical points, satisfying condition 1 is a subspace of $\mathcal{F}(M, P)$. On the other hand, if a smooth function $f: M \rightarrow P$ has only isolated critical points, then by theorem proved by P. T. Church and J. G. Timourian [1] and independently by O. Prishlyak [36], the local topological structure of level sets near any critical point can be realized by level sets of homogeneous polynomial without multiple factors. So the space $\mathcal{F}(M, P)$ consists of "generic" maps with "topologically generic" critical points, see [26, Section $3,4]$.

2.3. f-adapted manifolds. To state the results about homotopy properties of orbits and stabilizers for functions from the class $M$ on surfaces we need the notion of $f$-adapted manifolds.

Let $f: M \rightarrow P$ be a smooth function from $\mathcal{F}$ on a compact surface $M$. A connected component of a level set $f^{-1}(c), c \in P$, is also called a leaf of $f$. A leaf is called regular if it contains no critical points and critical otherwise.

Let $K$ be a (regular or critical) leaf of $f$. For $\varepsilon>0$ let $N_{\varepsilon}$ be a connected component of $f^{-1}[c-\varepsilon, c+\varepsilon]$ containing $K$. Then $N_{\varepsilon}$ is called an $f$-regular neighborhood of $K$ if $\varepsilon$ is so small that $N_{\varepsilon}-K$ contains no critical points and no boundary components.

A submanifold $X \subset M$ is called $f$-adapted if $X=\cup_{i=1}^{a} A_{i}$, where each $A_{i}$ is either a critical point of $f$, or a regular leaf of $f$, or an $f$-regular neighborhood of some (regular or critical) leaf of $f$. Note that if $X$ is an $f$-adapted subsurface, then $\left.f\right|_{X}: X \rightarrow P$ belongs to $\mathcal{F}(X, P)$, see [26].

For a set $X$ we denote by $|X|$ the number of point in $X$; if $X$ is an infinite set we put $|X|=\infty$.

2.4. Homotopy properties of orbits and stabilizers. The following theorem describes the general homotopy properties of orbits.

Theorem 2.4.1 ([20,24,25,39]). Let $f \in \mathcal{F}(M, P)$ be a function on a smooth compact surface $M$ and $X$ be an $f$-adapted submanifold. Then the following statements hold.

(1) The map

$$
\zeta_{f}: \mathcal{D}(M, X) \rightarrow \mathcal{O}(f, X), \quad \zeta_{f}(h)=f \circ h,
$$


is a locally trivial principal fibration with the fiber $\mathcal{S}(f, X)$. The restriction $\left.\zeta_{f}\right|_{\mathcal{D}_{\text {id }}(M, X)}: \mathcal{D}_{\text {id }}(M, X) \rightarrow \mathcal{O}_{f}(f, X)$ is also a locally trivial principal fibration with the fiber $\mathcal{S}^{\prime}(f, X)$. The orbit $\mathcal{O}_{f}(f, X)$ is a Frèchet manifold, so it has a homotopy type of a $C W$ complex.

(2) $\mathcal{O}_{f}(f, X)=\mathcal{O}_{f}(f, X \cup \partial M)$, and so

$$
\pi_{k} \mathcal{O}_{f}(f, X) \cong \pi_{k} \mathcal{O}_{f}(f, X \cup \partial M)
$$

for $k \geqslant 1$.

(3) Suppose that either $f$ has a saddle point or $M$ is non-orientable surface. Then $\mathcal{S}_{\text {id }}(f)$ is contractible, $\pi_{k} \mathcal{O}_{f}(f) \cong \pi_{k} M, k \geqslant 3, \pi_{2} \mathcal{O}_{f}(f)=0$, and for $\pi_{1} \mathcal{O}_{f}(f)$ the following short sequence of groups

$$
\pi_{1} \mathcal{D}_{\text {id }}(M) \stackrel{\zeta_{1}}{\longrightarrow} \pi_{1} \mathcal{O}_{f}(f) \stackrel{\partial_{1}}{\longrightarrow} \pi_{0} \mathcal{S}^{\prime}(f)
$$

is exact, where $\zeta_{1}$ is a homomorphism induced by $\zeta_{f}$ and $\partial_{1}$ is a boundary map for long exact sequence of the fibration $\zeta_{f}$. Moreover, $p\left(\pi_{1} \mathcal{D}_{\mathrm{id}}(M)\right)$ contains in the center of $\pi_{1} \mathcal{O}_{f}(f)$.

(4) If $\chi(M)<|X|$, then $\pi_{1} \mathcal{D}_{\text {id }}(M, X)$ is contractible (in particular, when $X$ is finite or when $\chi(M)<0), \pi_{k} \mathcal{O}_{f}(f, X)=0$ for $k \geqslant 2$ and the boundary map

$$
\pi_{1} \mathcal{O}_{f}(f, X) \stackrel{\partial_{1}}{\cong} \pi_{0} \mathcal{S}^{\prime}(f, X)
$$

is an isomorphism.

Notice that the sequence (2.3) is nonzero part of a long exact sequence of homotopy groups of the fibration $\zeta_{f}$. We briefly recall the definition of $\partial_{1}$. Let $\tilde{f}$ be a loop in $\mathcal{O}_{f}(f)$ based in $f: M \rightarrow$ P, i.e. $\tilde{f}:[0 ; 1] \rightarrow \mathcal{O}_{f}(f)$ with $\tilde{f}_{0}=\tilde{f}_{1}=f$. Then by Theorem 2.4.1(1) there exists a lifting of $\tilde{f}$, that is a path $h:[0 ; 1] \rightarrow \mathcal{D}_{\text {id }}(M)$ such that

$$
\tilde{f}_{t}=f \circ h_{t}, \quad h_{0}=\operatorname{id}_{M}, \quad h_{1}=h \in \mathcal{S}^{\prime}(f) .
$$

Denote by $[\tilde{f}]$ and $[h]$ the corresponding homotopy classes $\tilde{f}$ and $h$ in $\pi_{1} \mathcal{O}_{f}(f)$ and $\pi_{0} \mathcal{S}^{\prime}(f)$ respectively. Then the boundary homomorphism is defined by $\partial_{1}[\tilde{f}]=[h]$.

Moreover there is an isomorphism

$$
\varkappa: \pi_{1}\left(\mathcal{D}_{\mathrm{id}}(M), \mathcal{S}^{\prime}(f)\right) \longrightarrow \pi_{1} \mathcal{O}_{f}(f), \quad \varkappa:\left[\left\{h_{t}\right\}\right] \longmapsto\left[\left\{f \circ h_{t}\right\}\right],
$$

where $h_{t}: M \rightarrow M, t \in[0 ; 1]$ is an isotopy of $M$ with $h_{0}=\operatorname{id}_{M}$ and $h_{1} \in \mathcal{S}^{\prime}(f)$. 


\section{Auxiliary constructions}

3.1. Null-homotopic functions from $\mathcal{F}\left(M, S^{1}\right)$ and their orbits. A function $f: M \rightarrow S^{1}$ homotopic to a constant map will be called nullhomotopic.

It is easy to see that homotopy properties of null-homotopic functions from $\mathcal{F}\left(M, S^{1}\right)$ are the same as the ones of its "universal" lift, an ordinary function from $\mathcal{F}(M, \mathbb{R})$ arising from a universal cover of $S^{1}$. To be more precise, consider a universal covering map $p: \mathbb{R} \rightarrow S^{1}$ given by $p(t)=e^{2 \pi i t}$. Then by the lifting property for maps [11, Proposition 1.33], there exist a unique smooth function $\tilde{f}: M \rightarrow \mathbb{R}$ (up to a choice of appropriate triple of points) such that $p \circ \tilde{f}=f$. Note that the function $\tilde{f}$ is also a function from $\mathcal{F}(M, \mathbb{R})$. This function $\tilde{f}$ will be called a universal lift of $f$. The fact that homotopy properties of $\mathcal{O}_{f}(f)$ are the same as for $\mathcal{O}_{\tilde{f}}(\tilde{f})$ follows from the proposition.

Proposition 3.1.1. Let $f \in \mathcal{F}\left(M, S^{1}\right)$ be a null-homotopic function and $\tilde{f}: M \rightarrow \mathbb{R}$ be its universal lift. Then $\mathcal{O}(f)$ and $\mathcal{O}(\tilde{f})$ are homeomorphic, and hence $\mathcal{O}_{f}(f) \cong \mathcal{O}_{\tilde{f}}(\tilde{f})$.

Proof. Recall that $\zeta_{f}: \mathcal{D}(M) \rightarrow \mathcal{O}(f)$ and $\zeta_{\tilde{f}}: \mathcal{D}(M) \rightarrow \mathcal{O}(\tilde{f})$ are locally trivial principal fibrations with fibers $\mathcal{S}(f)$ and $\mathcal{S}(\tilde{f})$ respectively. Then $\zeta_{f}$ decomposes as the composition

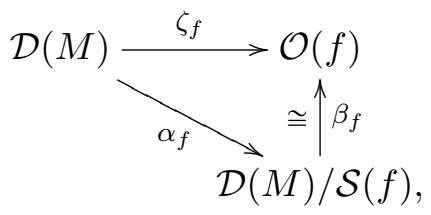

where $\alpha_{f}(h)=\bar{h}$ is an open quotient map, and $\beta_{f}(\bar{h})=\zeta_{f}(h)$ is an induced by $\zeta_{f}$ homeomorphism, where $\bar{h}$ is a coset of $h \in \mathcal{D}(M)$ modulo $\mathcal{S}(f)$. The same decomposition holds for $\zeta_{\tilde{f}}$. So $\mathcal{O}(f)$ and $\mathcal{O}(\tilde{f})$ are homeomorphic to quotient groups $\mathcal{D}(M) / \mathcal{S}(f)$ and $\mathcal{D}(M) / \mathcal{S}(\tilde{f})$ respectively. S. Maksymenko [27, Lemma 5.3] showed that if $f$ is null-homotopic then the stabilizers of $f$ and $\tilde{f}$ coincide: $\mathcal{S}(f) \cong \mathcal{S}(\tilde{f})$. Therefore the quotient spaces $\mathcal{D}(M) / \mathcal{S}(f)$ and $\mathcal{D}(M) / \mathcal{S}(\tilde{f})$ are the same as well, which implies that $\mathcal{O}(f)$ is homeomorphic to $\mathcal{O}(\tilde{f})$, and so $\mathcal{O}_{f}(f) \cong \mathcal{O}_{\tilde{f}}(\tilde{f})$.

By Proposition 3.1.1 homotopy properties of $\mathcal{O}_{f}(f)$ for a null-homotopic function $f \in \mathcal{F}\left(M, S^{1}\right)$ are the "same" as for $\mathcal{O}_{\tilde{f}}(\tilde{f})$ where $\tilde{f}$ is a universal 
lift of $f$. Therefore this case is completely reduced to the real-valued case, and so we will focus on the case when $f$ is not null-homotopic.

3.2. Graphs of $P$-valued Morse functions. Kronrod-Reed graphs are important tools for study of smooth functions since they carry a lot of informations about its combinatorial structure. We recall this definition. Let $f \in \mathcal{F}(M, P)$ be a function on a smooth compact oriented surface $M$ and $c \in P$. Let $\Xi$ be a partition of $M$ into connected components of level sets (leaves, see subsection 2.3) of $f$. It is well-known that the quotient space $M / \Xi$ denoted by $\Gamma_{f}$ has a structure of an 1-dimensional CW complex called a Kronrod-Reeb graph of $f$, or simply a graph of $f$ [38].

Let $q_{f}: M \rightarrow \Gamma_{f}$ be a quotient map. Then the map $f: M \rightarrow P$ can be presented as the composition of the projection $q_{f}$ onto $\Gamma_{f}$ and the map $f_{\Gamma}$ induced by $f$ :

$$
f=f_{\Gamma} \circ q_{f}: \quad M \stackrel{q_{f}}{\longrightarrow} \Gamma_{f} \stackrel{f_{\Gamma}}{\longrightarrow} P .
$$

3.3. Graphs of $S^{1}$-valued function on $T^{2}$. Graphs of ordinary Morse functions on $T^{2}$ were studied in [16]. For functions with isolated singularities on 2-torus the following lemma holds true.

Lemma 3.3.1 (cf. Lemma $3.1[6]$ ). Let $f: T^{2} \rightarrow S^{1}$ be a function with isolated singularities.

(1) The map $q_{f}^{*}: \pi_{1} T^{2} \rightarrow \pi_{1} \Gamma_{f}$ induced by $q_{f}$ is an epimorphism with a non-zero kernel. So $b_{1}\left(\Gamma_{f}\right) \leqslant 1$, i.e. $\Gamma_{f}$ can be either a tree or contains a unique cycle.

(2) If $f$ is not null-homotopic, then $\Gamma_{f}$ is not a tree.

It is well-known that the inequality $b_{1}\left(\Gamma_{f}\right) \leqslant 1$ holds for generic circlevalued Morse functions on 2-torus [2, Theorem 3.7] and for arbitrary circlevalued Morse functions on 2-torus this inequality follows from Morse form foliation theory, see [9, Theorem 2.1, Theorem 3.1, Example 3.2].

Proof of Lemma 3.3.1. (1) This statement for ordinary Morse functions is proved in [6, Lemma 3.1] and holds true for functions with isolated singularities. The reader can verify it step-by-step as in [6, Lemma 3.1].

(2) Assume the converse holds and consider the sequence of fundamental groups induced by (3.1):

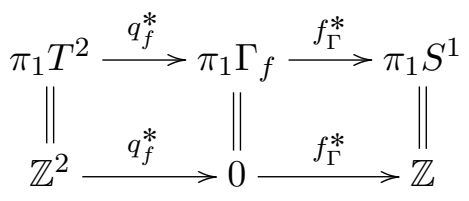


The homomorphism $f_{\Gamma}^{*}$ is a zero-map, so the homomorphism $f^{*}=f_{\Gamma}^{*} \circ q_{f}^{*}$ induced by $f$ is also a zero map. This leads to the contradiction that $f$ is not null-homotopic, so $\Gamma_{f}$ contains a cycle.

The case of functions $f: T^{2} \rightarrow P$ from the class $\mathcal{F}$ whose graphs are trees is a special case: it is only possible when $f$ is null-homotopic. So in what follows we will study only the case of functions whose graphs contain a cycle. Examples of such functions are given in Figure 3.1. Note that functions in
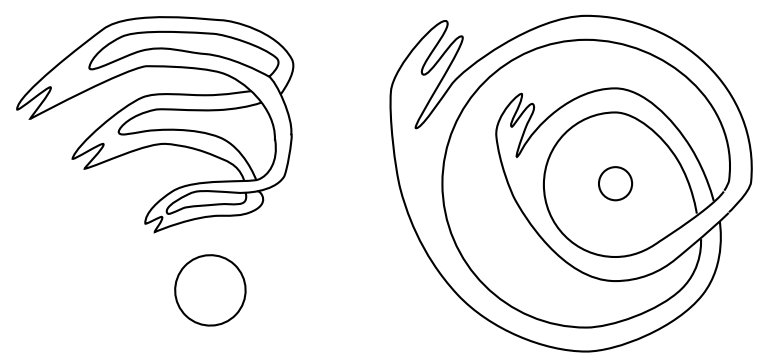

FIGURE 3.1. Null-homotopic (left) and not null-homotopic (right) functions $T^{2} \rightarrow S^{1}$

Figure 3.1 have certain "combinatorial symmetries" preserving the given functions which play an essential role in the description of $\pi_{1} \mathcal{O}_{f}(f)$.

3.4. Wreath products. To state our main result we need a notion of wreath product of groups of a special kind. Let $G$ be a group and $n \geqslant 1$ be an integer. A semi-direct product $G^{n} \rtimes \mathbb{Z}$ with respect to a non-effective $\mathbb{Z}$-action $\alpha$ on $G^{n}$ by cyclic shifts

$$
\alpha\left(b_{0}, b_{1}, \ldots, b_{n-1} ; k\right)=\left(b_{k}, b_{1+k}, \ldots, b_{n+k-1}\right),
$$

where all indexes are taken modulo $n$, will be denoted by $G \imath_{n} \mathbb{Z}$ and called a wreath product of $G$ with $\mathbb{Z}$ under $n$. Notice that this definition differs from the standard one: it corresponds to a non-effective action of $\mathbb{Z}$ on the set $\mathbb{Z}_{n}$ by cyclic shifts, while wreath products are usually studied for effective actions, [31].

\section{MAin RESUlT}

The following theorem describes an algebraic structure of $\pi_{1} \mathcal{O}_{f}(f)$ for functions $f: T^{2} \rightarrow P$ from the class $\mathcal{F}$ whose graphs contain a cycle. It will be proved in Section 6 . 
Theorem 4.1. Let $f$ be a function from $\mathcal{F}\left(T^{2}, P\right)$ with at least one critical point and whose graph $\Gamma_{f}$ contains a cycle. Then there exist a cylinder $Q \subset T^{2}$ and $n \in \mathbb{N}$ such that $\left.f\right|_{Q} \in \mathcal{F}(Q, P)$ and there is an isomorphism

$$
\pi_{1} \mathcal{O}_{f}(f) \cong \pi_{0} \mathcal{S}^{\prime}\left(\left.f\right|_{Q}, N(\partial Q)\right) \imath_{n} \mathbb{Z},
$$

where $N(\partial Q)$ is some $f$-regular neighborhood of $\partial Q$.

Remark 4.2. Theorem 4.1 generalizes the main result of the paper [29]. To prove [29, Theorem 1.6] we mainly use "local" technique, i.e. properties of diffeomorphisms of subsurfaces of $T^{2}$ and "glue" them together to obtain global ones. So Theorem 4.1 can be proved step-by-step by the same arguments and strategy. In the present paper we give a more straightforward proof of this result separating algebraic aguments from topological ones. Proofs of some known facts will be given only for the sake of completeness.

\section{Additional CONStruCtions And DEFinitions}

5.1. Curves on $T^{2}$. Let $f$ be a function from $\mathcal{F}\left(T^{2}, P\right)$ whose graph $\Gamma_{f}$ contains a unique cycle denoted by $\Lambda$, let also $q_{f}: T^{2} \rightarrow \Gamma_{f}$ be a projection induced by $f$. Let $z$ be a point in $\Lambda, c=f\left(q_{f}^{-1}(z)\right)$ be a point in $S^{1}$, and $C$ be a regular leaf (connected component) of $f^{-1}(c)$. Note that $f^{-1}(c)$ consists of finitely many connected components and it is invariant under the action of $\mathcal{S}^{\prime}(f)$. Put $\mathcal{C}:=\left\{h(C) \mid h \in \mathcal{S}^{\prime}(f)\right\}$. Then $\mathcal{C}$ consists of finitely many mutually disjoint and non-separating simple closed curves on $T^{2}$. Hence we can cyclically enumerate those curves

$$
\mathcal{C}=\left\{C_{0}=C, C_{1}, C_{2}, \ldots, C_{n-1}\right\}
$$

for some $n \in \mathbb{N}$, so that each consecutive pair $C_{i}$ and $C_{i+1}$ bounds a cylinder $Q_{i} \subset T^{2}$ and those cylinders mutually disjoint, where all indexes are taken modulo $n$.

5.2. $f$-regular neighborhoods of curves. Regard $S^{1}$ and $T^{2}$ as quotient spaces $\mathbb{R} / \mathbb{Z}$ and $\mathbb{R}^{2} / \mathbb{Z}^{2}$ respectively. By a proper choose of coordinates on $T^{2}$ one can assume that the following conditions hold:

- $C_{i}=\left\{\frac{i}{n}\right\} \times S^{1} \subset \mathbb{R}^{2} / \mathbb{Z}^{2}=T^{2}$, so we can regard each curve $C_{i}$ as a meridian of $T^{2}$, and the curve $C^{\prime}=\{0\} \times S^{1}$ as a longitude of $T^{2}$;

- there exists $\varepsilon>0$ such that for all $t \in\left(\frac{i}{n}-\varepsilon ; \frac{i}{n}+\varepsilon\right)$ the curve $\{t\} \times S^{1}$ is a regular leaf of some level set of $f$.

This assumption makes possible to define $f$-regular neighborhoods of curves from $\mathcal{C}$, see Subsection 2.3. So an $f$-regular neighborhood $V$ of a curve $C$ is saturated neighborhood which has a cylindrical structure.

In this place we fix two families of $f$-regular neighborhoods $V_{i}$ and $W_{i}$ of $C_{i}, i=0, \ldots, n-1$, needed for further exposition, so that $V_{i} \cap V_{j}=\varnothing$, 
$V_{i} \subset \operatorname{Int}\left(W_{i}\right)$ for $i \neq j$, and for each $i, j$ there exists $h \in \mathcal{S}^{\prime}(f)$ such that $h\left(V_{i}\right)=V_{j}$. In particular, the unions $\mathrm{V}=\bigcup_{i=0}^{n-1} V_{i}$ and $\mathrm{W}=\bigcup_{i=0}^{n-1} W_{i}$ are $\mathcal{S}^{\prime}(f)$-invariant.

5.3. Generators of $\pi_{1} \mathcal{D}_{\text {id }}\left(T^{2}\right)$. Let $\mathbf{L}, \mathbf{M}: T^{2} \times[0 ; 1] \rightarrow T^{2}$ be two isotopies defined by

$$
\mathbf{L}(x, y, t)=(x+t \bmod 1, y), \quad \mathbf{M}(x, y, t)=(x, y+t \bmod 1)
$$

for $x \in C^{\prime}, y \in C_{k}, k=0,1, \ldots, n-1$. Geometrically $\mathbf{L}$ is a rotation of $T^{2}$ along its longitude, and $\mathbf{M}$ is a rotation along meridians. The isotopies $\mathbf{L}$ and $\mathbf{M}$ can be regarded as loops in $\mathcal{D}_{\text {id }}\left(T^{2}\right)$. Evidently, $\mathbf{L}$ and $\mathbf{M}$ commute and it is well known that $\pi_{1} \mathcal{D}_{\text {id }}\left(T^{2}\right)=\langle\mathbf{L}\rangle \times\langle\mathbf{M}\rangle$, see $[4,10]$.

5.4. Dehn twists and slides along curves from $\mathcal{C}$. Let $Q=S^{1} \times[-1 ; 1]$ be a cylinder and $C$ be the curve $S^{1} \times\{0\}$, and $\alpha, \beta:[-1 ; 1] \rightarrow[0 ; 1]$ be two smooth functions such that

$$
\alpha(x)=\left\{\begin{array}{ll}
0, & x \in[-1 ;-1 / 2], \\
1, & x \in[1 / 2 ; 1],
\end{array} \quad \beta(x)= \begin{cases}0, & x \in[-1 ;-2 / 3] \cup[2 / 3 ; 1], \\
1, & x \in[-1 / 3 ; 1 / 3] .\end{cases}\right.
$$

Define two diffeomorphisms of $Q$ by formulas:

$$
\tau(z, t)=\left(z e^{\alpha(t)}, t\right) \quad \theta(z, t)=\left(z e^{\beta(t)}, t\right), \quad(z, t) \in Q ;
$$

the diffeomorphisms $\tau$ and $\theta$ are called a Dehn twist and a slide along $C=S^{1} \times\{0\}$. Note that $\tau$ is fixed on some neighborhood of $\partial Q$, and $\theta$ is fixed on some neighborhood of $C \cup \partial Q$. A diffeomorphism of a smooth surface $M$ supported in some cylindrical neighborhood of a simple closed and two-sided curve $C \subset M$ isotopic to a Dehn twist with respect to the boundary of this neighborhood will be called a Dehn twist along $C$ on $M$. Similarly the notion of slide along $C$ can be extended to the case of surfaces.

Recall that a vector field $F$ on a smooth oriented surface $M$ is called Hamiltonian-like for a function $f$ from the class $\mathcal{F}$ if the following conditions satisfied.

- The set of zeros of $F$ coincides with the set of critical points of $f$.

- $f$ is constant along $F$.

- Let $z$ be a critical point of $f$. Then there exists a local coordinate system $(x, y)$ such that $f(z)=0, f(x, y)= \pm x^{2} \pm y^{2}$ near $z$, and in this coordinates $F$ has the form $F(x, y)=-f_{y}^{\prime} \partial / \partial x+f_{x}^{\prime} \partial / \partial y$.

Fix a Hamiltonian-like vector field $F$ of the given function $f: T^{2} \rightarrow S^{1}$, and let $\mathbf{F}_{t}: T^{2} \rightarrow T^{2}, t \in \mathbb{R}$ be its flow. The set $\mathbf{W}$ does not contain zeros of $\mathbf{F}$ and it is $\mathbf{F}$-invariant and consists of periodic orbits. Therefore one can additionally assume that the periods of all trajectories of $\mathbf{F}$ on $\mathbf{W}$ equal 1. 
Denote by $\theta_{i}: T^{2} \rightarrow T^{2}$ the slide along the curve $C_{i}$ supported in $W_{i} \backslash V_{i}$, $i=0,1, \ldots, n-1$, and let $\theta=\theta_{0} \circ \theta_{1} \circ \ldots \circ \theta_{n-1}$. We proved that there exists a smooth function $\sigma: T^{2} \rightarrow \mathbb{R}$ which satisfies

- $\sigma$ is constant along trajectories of $\mathbf{F}$,

- $\sigma=1$ on $\mathrm{V}, \sigma=0$ on $T^{2} \backslash \mathrm{W}$, and

- $\theta=\mathbf{F}_{\sigma}$,

and therefore $\theta^{k}=\mathbf{F}_{k \sigma}$, see [29, Lemma 5.2]. A free abelian group generated by $\theta$ will be denoted by $\langle\theta\rangle$.

5.5. Characterization of direct and wreath products. In this paragraph we recall conditions when the group $G$ splits into a direct product of its subgroups and discuss when $G$ splits into a wreath product as in subsection 3.4. Let $G$ be a group and $G_{1}, \ldots, G_{n}$ be their subgroups. Then $G$ is said to split into a direct product $G_{1} \times G_{2} \times \ldots \times G_{n}$ whenever the following three conditions satisfied:

(D1) $G_{i} \cap G_{j}=\{e\}$, for $i \neq j=1,2, \ldots, n$, where $e$ is the unit of $G$,

(D2) $G_{i} G_{j}=G_{j} G_{i}$ for all $i, j=1,2, \ldots, n$,

(D3) groups $G_{1}, G_{2}$ and $G_{n}$ generate $G$.

The following lemma gives conditions when the group $G$ splits into a wreath product $L_{0} 2_{n} \mathbb{Z}$ for some subgroup $L_{0} \subset G$.

Lemma 5.5.1 (Lemma 2.3 [27]). Let $\phi: G \rightarrow \mathbb{Z}$ be an epimorphism and $L_{0}$ be a subgroup of $\operatorname{ker} \phi$ Let also $g \in G$ be such that $\phi(g)=1$. Assume also that for some $n \in \mathbb{Z}$ the following conditions hold:

(1) $g^{n}$ commutes with $\operatorname{ker} \phi$,

(2) $\operatorname{ker} \phi$ splits into a direct product of

$$
L_{0}, \quad L_{1}=g^{-1} L_{0} g^{1}, \quad \ldots, \quad L_{m-1}=g^{-(n-1)} L_{0} g^{n-1} .
$$

Then the map $\xi: L_{0} \imath_{n} \mathbb{Z} \rightarrow G$ given by the formula

$$
\begin{aligned}
\xi\left(b_{0}, b_{1}, \ldots, b_{n-1}, k\right) & =b_{0}\left(g^{-1} b_{1} g^{1}\right)\left(g^{-2} b_{2} g^{2}\right) \ldots\left(g^{-n+1} b_{n-1} g^{n-1}\right) g^{k} \\
& =b_{0} g^{-1} b_{1} \ldots g^{-1} b_{n-1} b^{-1+n+k}
\end{aligned}
$$

is an isomorphism.

\section{Proof of Theorem 4.1}

6.1. Structure of the proof. Our main proof-tool is Lemma 5.5.1. So we need to define a data of a "natural" epimorphism $\phi: \pi_{1} \mathcal{O}_{f}(f) \rightarrow \mathbb{Z}$, an element $g$ from ker $\phi$, and groups $L_{i}, i=0,1, \ldots, n-1$, such in Lemma 5.5.1. 
6.2. Epimorphism $\phi$ and its kernel. Let $V_{i}$ and $W_{i}$ be fixed $f$-regular neighborhoods of $C_{i} \in \mathcal{C}, i=0,1, \ldots, n-1$, such in Subsection 5.2.

Proposition 6.2.1 (Theorem $6.1[29]$ ). There exists an epimorphism

$$
\phi: \pi_{1} \mathcal{O}_{f}(f) \rightarrow \mathbb{Z}
$$

whose kernel is isomorphic to $\pi_{0} \mathcal{S}^{\prime}(f, \mathrm{~W})$, i.e. the following sequence of groups

$$
\pi_{0} \mathcal{S}^{\prime}(f, \mathrm{~W}) \longleftrightarrow \pi_{1} \mathcal{O}_{f}(f) \stackrel{\phi}{\longrightarrow} \mathbb{Z}
$$

is exact.

Proof. This result is proved in [29], but for completeness of our exposition we will recall the construction of an epimorphism $\phi$. Let

$$
q: \mathbb{R} \times S^{1} \rightarrow S^{1} \times S^{1}=T^{2}
$$

be a covering map given by $q(x, y)=\left(\frac{x}{n} \bmod 1, y\right)$. Then

$$
q\left(\{i\} \times S^{1}\right)=C_{i \bmod n}, i \in \mathbb{Z}, \quad q^{-1}(\mathcal{C})=\mathbb{Z} \times S^{1} .
$$

Let $\omega:[0 ; 1] \rightarrow \mathcal{O}_{f}(f)$ be a loop and $h: T^{2} \times[0 ; 1] \rightarrow T^{2}$ be an isotopy such that $\omega_{t}=f \circ h_{t}$ and $h_{0}=\operatorname{id}_{T^{2}}, h_{1} \in \mathcal{S}^{\prime}(f)$. There exist an isotopy

$$
\tilde{h}:\left(\mathbb{R} \times S^{1}\right) \times[0 ; 1]=\mathbb{R} \times S^{1}
$$

such that $\tilde{h}_{0}=\operatorname{id}_{\mathbb{R} \times S^{1}}$ and $q \circ \tilde{h}_{t}=h_{t} \circ q$ for all $t \in[0 ; 1]$. Since $h_{1}(\mathcal{C})=\mathcal{C}$, it follows from the definition of $q$ that $\tilde{h}_{1}\left(\mathbb{Z} \times S^{1}\right)=\mathbb{Z} \times S^{1}$. Then there exists an integer $\phi_{h}$ such that

$$
\tilde{h}_{1}\left(\{i\} \times S^{1}\right)=\left\{i+\phi_{h}\right\} \times S^{1} .
$$

The number $\phi_{h}$ depends only on the homotopy class of $h$, so on the isotopy class of the loop $\omega$, and the correspondence

$$
[\omega] \stackrel{\phi}{\longrightarrow} \phi_{h}
$$

defined by (6.1) is an epimorphism $\phi: \pi_{1} \mathcal{O}_{f}(f) \rightarrow \mathbb{Z}$.

Evidently, the kernel of $\phi$ consists of homotopy classes of isotopies

$$
h: T^{2} \times[0 ; 1] \rightarrow T^{2}
$$

such that $h_{1}$ leaves invariant each curve $C_{i}$ from $\mathcal{C}$, i.e. $h_{1}\left(C_{i}\right)=C_{i}$. It was shown [20, Lemma 4.14] that each such $h_{1}$ can be isotoped in $\mathcal{S}^{\prime}(f)$ to the diffeomorphism $h_{1}^{\prime}$ which is fixed on an $f$-cylindrical neighborhood W of $\mathcal{C}$, so $h_{1}^{\prime} \in \mathcal{S}^{\prime}(f, \mathrm{~W})$. By (4) of Theorem 2.4.1 and equation (2.4) the composition

$$
\pi_{1}\left(\mathcal{D}_{\mathrm{id}}\left(T^{2}, \mathrm{~W}\right), \mathcal{S}^{\prime}(f, \mathrm{~W})\right) \underset{\varkappa^{-1}}{\stackrel{\longrightarrow}{\cong}} \pi_{1} \mathcal{O}_{f}(f, \mathrm{~W}) \stackrel{\partial}{\cong} \pi_{0} \mathcal{S}^{\prime}(f, \mathrm{~W})
$$


is an isomorphism. So the kernel of $\phi$ is isomorphic to $\pi_{0} \mathcal{S}^{\prime}(f, \mathrm{~W})$.

6.3. Special isotopy and subgroups of $\operatorname{ker} \phi$. The following proposition holds true.

Proposition 6.3.1 (Theorem 6.1 (c), [29]). There exist an isotopy

$$
g: T^{2} \times[0 ; 1] \rightarrow T^{2}
$$

satisfying the following conditions

(1) $g_{1} \in \mathcal{S}^{\prime}(f, \mathrm{~W})$,

(2) $g_{1}^{n}=\operatorname{id}_{T^{2}}$,

(3) $g_{1}\left(Q_{i}\right)=Q_{i+1}, i=0,1, \ldots, n-1$,

(4) $\phi\left(\left[f \circ g_{t}\right]\right)=1$.

Proof. By definition of the set $\mathcal{C}$, there exists $g_{1} \in \mathcal{S}^{\prime}(f)$ such that

$$
g\left(Q_{i}\right)=Q_{i+1}
$$

which proves (3). To show (1) and (2) we need to change $g$ with $\sigma=\mathbf{L}_{1 / n}$ on $\mathbf{W}$ in $\mathcal{S}^{\prime}(f)$, where $\mathbf{L}$ is defined in (5.1); this was done in (c) of [29, Theorem 6.1]. The resulting diffeomorphism will be denoted by $g_{1}$.

(4) Let $g: T^{2} \times[0 ; 1] \rightarrow T^{2}$ be an isotopy between $g_{1}$ and $\mathrm{id}_{T^{2}}$. So $\phi([g])=a n+1$ for some $n \in \mathbb{Z}$. If $a \neq 0$ then we replace $\left[g_{t}\right]$ by $\left[g_{t} \circ \mathbf{L}_{t}^{-a}\right]$ in order to have $\phi\left(\left[f \circ g_{t}\right]\right)=1$.

Denote by $X_{i}^{-}$and $X_{i}^{+}$the following intersections $Q_{i} \cap W_{i}, Q_{i} \cap W_{i+1}$, and set $X_{i}=X_{i}^{-} \cup X_{i}^{+}, U_{i}=Q_{i} \backslash X_{i}$ for $i=0,1, \ldots, n-1$. The set $X_{i}$ is an $f$-adapted neighborhood of the boundary $\partial Q_{i}$ of the cylinder $Q_{i} \subset T^{2}$, see Figure 6.1.

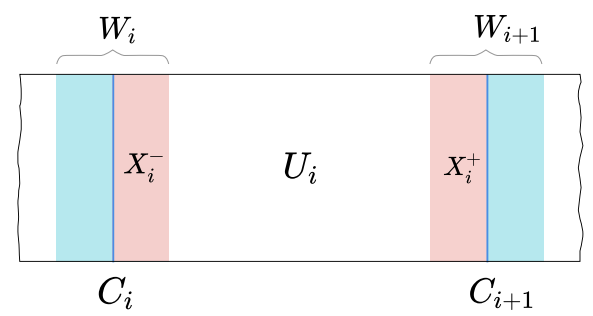

Figure 6.1.

Define the following subgroup $L_{i}^{\prime}$ of the kernel of $\phi$ :

$$
L_{i}^{\prime}=\pi_{0} \mathcal{S}^{\prime}\left(f, T^{2}-U_{i}\right), \quad i=0,1, \ldots, n-1 .
$$


By definition, the elements of $L_{i}^{\prime}$ are isotopy classes of diffeomorphisms supported on $U_{i}$. Let $g: T^{2} \times[0 ; 1] \rightarrow T^{2}$ be an isotopy from Proposition 6.3.1. Since $g_{1}$ satisfies (1)-(4) of Proposition 6.3.1, it follows that

$$
L_{i}^{\prime}=\left[g_{1}^{-i}\right] L_{0}^{\prime}\left[g_{1}^{i}\right], \quad i=0,1, \ldots, n-1 .
$$

Each diffeomorphism $h \in \mathcal{S}^{\prime}\left(f, T^{2} \backslash U_{i}\right)$ is fixed on $T^{2} \backslash U_{i}$, so the restriction $\left.h \rightarrow h\right|_{Q_{i}}$ induces an isomorphism

$$
\beta_{i}: \pi_{0} \mathcal{S}^{\prime}\left(f, T^{2} \backslash U_{i}\right) \rightarrow \pi_{0} \mathcal{S}^{\prime}\left(\left.f\right|_{Q_{i}}, X_{i}\right)
$$

given by the formula $\beta_{i}([h])=\left[\left.h\right|_{Q_{i}}\right]$. Let $h_{i}$ be a diffeomorphism from $\mathcal{S}^{\prime}\left(\left.f\right|_{Q_{i}}, X_{i}\right)$, and $h_{i}^{\prime}$ be its extension to $T^{2}$ by the identity map. Then the inverse of $\beta_{i}$ is given by the rule: $\beta_{i}^{-1}\left(\left[h_{i}\right]\right)=\left[h_{i}^{\prime}\right]$. So we will not distinguish groups $\pi_{0} \mathcal{S}^{\prime}\left(f, T^{2} \backslash U_{i}\right)$ and $\pi_{0} \mathcal{S}^{\prime}\left(\left.f\right|_{Q_{i}}, X_{i}\right)$, and we set $L_{i}=\pi_{0} \mathcal{S}^{\prime}\left(\left.f\right|_{Q_{i}}, X_{i}\right)$.

6.4. The end of the proof. The following lemma completes the proof.

Lemma 6.4.1. The data of an epimorphism $\phi$ and the element $g$ from Proposition 6.3.1, and groups $L_{i}=\pi_{0} \mathcal{S}^{\prime}\left(\left.f\right|_{Q_{i}}, X_{i}\right)$ from (6.2) satisfy conditions of Lemma 5.5.1. So $\pi_{1} \mathcal{O}_{f}(f)$ is isomorphic to $\pi_{0} \mathcal{S}^{\prime}\left(\left.f\right|_{Q_{0}}, X_{0}\right) \ell_{n} \mathbb{Z}$ and this isomorphism is given by the formula (5.2).

Proof. Let $g: T^{2} \times[0 ; 1] \rightarrow T^{2}$ is an isotopy defined in Proposition 6.3.1. By (1) of Proposition 6.3.1 the diffeomorphism $g_{1}^{n}=\mathrm{id}_{T^{2}}$, and so it commutes with ker $\phi$. Thus (1) of Lemma 5.5.1 holds true.

To verify (1) of Lemma 5.5.1 we need to check that the three conditions (D1)-(D3) from subsection 5.5 satisfied:

(D1) $L_{i} \cap L_{j}=\left[\mathrm{id}_{T^{2}}\right], i \neq j$;

(D2) $L_{i} L_{j}=L_{j} L_{i}$;

(D3) groups $L_{0}, L_{1}, \ldots, L_{n-1}$ generate $\operatorname{ker} \phi$;

for all $i, j=0,1, \ldots, n-1$. Conditions (D1) and (D2) follow from the fact that

$$
\operatorname{supp}\left(h_{i}\right) \cap \operatorname{supp}\left(h_{j}\right)=\varnothing
$$

for $\left[h_{i}\right] \in L_{i},\left[h_{j}\right] \in L_{j}, i \neq j=0,1, \ldots, n-1$.

Suppose [h] belongs to $\operatorname{ker} \phi$. Then $\left.h\right|_{Q_{i}} \in \mathcal{S}^{\prime}\left(\left.f\right|_{Q_{i}}, X_{i}\right)$ and there is a unique decomposition

$$
[h]=\left[\left.h\right|_{Q_{0}}\right]\left[\left.h\right|_{Q_{1}}\right] \ldots\left[\left.h\right|_{Q_{n-1}}\right] .
$$

On the other hand let $h_{i}$ be a diffeomorphism belonging to $\mathcal{S}^{\prime}\left(\left.f\right|_{Q_{i}}, X_{i}\right)$, $i=0,1, \ldots, n-1$. Then

$$
\beta_{0}^{-1}\left(\left[h_{0}\right]\right) \circ \beta_{1}^{-1}\left(\left[h_{1}\right]\right) \circ \ldots \circ \beta_{n-1}^{-1}\left(\left[h_{n-1}\right]\right)
$$


represents an element of ker $\phi$. So (D3) is true $i . e$ groups $L_{0}, L_{1}, \ldots, L_{n-1}$ generate $\operatorname{ker} \phi$.

\section{ThE KERNEL OF $\phi$ AND AN ISOTOPY $\mathbf{M}$}

Note that the group $\langle\mathbf{M}\rangle$ is contained in the kernel of $\phi$, however for the proof of Theorem 4.1 we do not need to know explicit forms of elements from ker $\phi$ representing elements of $\mathbf{M}$. Let $\mathrm{V}$ and $\mathrm{W}$ be fixed in subsection $5.2 f$-regular neighborhoods of $\mathcal{C}$. The following proposition describes the relationship between $\operatorname{ker} \phi$ and the group $\langle\mathbf{M}\rangle$.

Proposition 7.1. There exists an isomorphism

$$
\pi_{0} \mathcal{S}^{\prime}(f, \mathrm{~W}) \cong\langle\mathbf{M}\rangle \times \pi_{0} \mathcal{S}^{\prime}(f, \mathrm{~V}) .
$$

Proof. In [29] we showed that the isotopy class of $\mathbf{M}$ can be represented as the isotopy class of

$$
\theta=\theta_{0} \circ \theta_{1} \circ \ldots \circ \theta_{n-1}
$$

where $\theta_{i}$ is a slide along $C_{i}$ supported in $W_{i} \backslash V_{i}, i=0,1,2 \ldots, n-1$.

To prove this proposition we need to check that conditions (D1)-(D3) from subsection 5.5 hold for subgroups $\langle\theta\rangle$ and $\pi_{0} \mathcal{S}^{\prime}(f, \mathrm{~V})$ of $\pi_{0} \mathcal{S}^{\prime}(f, \mathrm{~W})$. Conditions (D1) and (D2) obviously hold since

$$
\operatorname{supp}(\theta) \cap \operatorname{supp}(h)=\varnothing
$$

for all $h \in \mathcal{S}^{\prime}(f, \mathrm{~V})$. Therefore it remains to show that for each $h$ in $\mathcal{S}^{\prime}(f, \mathrm{~W})$ there is a unique decomposition

$$
[h]=\left[\theta^{k(h)}\right] \circ\left[h^{\prime}\right],
$$

where $h^{\prime} \in \mathcal{S}^{\prime}(f, \mathrm{~V})$ and $k(h) \in \mathbb{Z}$. To define this decomposition we will use the same arguments such in [6, Theorem 5.5].

Let $h$ be a diffeomorphism from $\mathcal{S}^{\prime}(f, \mathrm{~W})$. Since $f$ is fixed on $\mathbf{W}$, it follows from [27, Lemma 6.1], that there exists a smooth function $\alpha: \mathrm{W} \rightarrow \mathbb{R}$ such that $h=\mathbf{F}_{\alpha}$. The restriction of $h$ and $\alpha$ onto $W_{i}$ are denoted by $h_{i}$ and $\alpha_{i}$ respectively. Since periods of all trajectories of $\left.\mathbf{F}\right|_{\mathrm{w}}$ are equal to 1 and $h$ is fixed on $\mathbf{W}$, it follows that the restriction $\left.\alpha_{i}\right|_{W_{i}}$ is constant and equals to some integer number $k_{i}(h) \in \mathbb{Z}$ (being an integer multiple of periods). The diffeomorphism $\left.h\right|_{Q_{i}}$ is isotopic relative $\mathrm{W} \cap Q_{i}$ to a Dehn twist $\tau^{a_{i}}$ supported on $\mathrm{W} \cap Q_{i}$, where $a_{i}=\alpha\left(C_{i+1}\right)-\alpha\left(C_{i}\right), i=0,1, \ldots, n-1$. Since $h \in \mathcal{S}^{\prime}(f, \mathrm{~W})$, it follows that $\left.h\right|_{Q_{i}}$ is isotopic to the identity map of $Q_{i}$. Hence

$$
a_{i}=\alpha\left(C_{i+1}\right)-\alpha\left(C_{i}\right)=k_{i+1}(h)-k_{i}(h)=0 .
$$

In other words, the numbers $k_{i}(h)$ pairwise coincide, and we will denote their common value by $k(h)$. 
Define an isotopy $H^{t}: T^{2} \rightarrow T^{2}$ between $h$ and $\theta^{-k(h)} \circ h$ by the formula

$$
H^{t}(h)=\mathbf{F}_{t k(h) \sigma}^{-1} \circ h .
$$

Notice that the diffeomorphism $H^{t}(h)$ is fixed on $\mathrm{V}$ for all $t \in[0 ; 1]$, and we have the following decomposition

$$
[h]=\left[\theta^{k(h)}\right] \circ\left[\theta^{-k(h)} \circ h\right]=\left[\theta^{k(h)}\right] \circ\left[H^{1}(h)\right]
$$

which coincides with (7.1) for $h^{\prime}=H^{1}(h)$.

\section{REFERENCES}

[1] P. T. Church, J. G. Timourian. Differentiable open maps of $(p+1)$-manifold to p-manifold. Pacific J. Math., 48:35-45, 1973, http://projecteuclid.org/euclid.pjm/ 1102945698.

[2] K. de Rezende, G. Ledesma, O. Manzoli-Neto, G. Vago. Lyapunov graphs for circle valued functions. Topology Appl., 245:62-91, 2018, doi: 10.1016/j.topol.2018.06.008.

[3] C. J. Earle, J. Eells. The diffeomorphism group of a compact Riemann surface. Bull. Amer. Math. Soc., 73:557-559, 1967, doi: 10.1090/S0002-9904-1967-11746-4.

[4] C. J. Earle, J. Eells. A fibre bundle description of Teichmüller theory. J. Differential Geometry, 3:19-43, 1969, doi: 10.4310/jdg/1214428816.

[5] C. J. Earle, A. Schatz. Teichmüller theory for surfaces with boundary. J. Differential Geometry, 4:169-185, 1970, doi: 10.4310/jdg/1214429381.

[6] B. Feshchenko. Deformations of functions on 2-torus. Proceedings of the International Geometry Center, 12(3):30-50, 2019, doi: 10.15673/tmgc.v12i3.1528.

[7] B. Feshhenko. Deformations of smooth functions on 2-torus, whose KR-graph is a tree. Proceedings of Institute of Mathematics of NAS of Ukraine, 12(6):22-40, 2015.

[8] K. Fukaya, Y.-G. Oh, H. Ohta, K. Ono. Lagrangian intersection Floer theory: anomaly and obstruction. Part I, volume 46 of AMS/IP Studies in Advanced Mathematics. American Mathematical Society, Providence, RI; International Press, Somerville, MA, 2009, doi: 10.1090/crmp/049/07.

[9] I. Gelbukh. On the structure of a Morse form foliation. Czechoslovak Math. J., 59(134)(1):207-220, 2009, doi: 10.1007/s10587-009-0015-5.

[10] A. Gramain. Le type d'homotopie du groupe des difféomorphismes d'une surface compacte. Ann. Sci. École Norm. Sup. (4), 6:53-66, 1973, doi: 10.24033/asens.1242.

[11] A. Hatcher. Algebraic topology. Cambridge University Press, Cambridge, 2002.

[12] M. Hutchings, Yi-Jen Lee. Circle-valued Morse theory and Reidemeister torsion. Geom. Topol., 3:369-396, 1999, doi: 10.2140/gt.1999.3.369.

[13] M. Hutchings, Yi-Jen Lee. Circle-valued Morse theory, Reidemeister torsion, and Seiberg-Witten invariants of 3-manifolds. Topology, 38(4):861-888, 1999, doi: 10.1016/S0040-9383(98)00044-5.

[14] K. Ikegami, O. Saeki. Cobordism group of Morse functions on surfaces. J. Math. Soc. Japan, 55(4):1081-1094, 2003, doi: 10.2969/jmsj/1191418765.

[15] B. Kalmár. Cobordism group of Morse functions on unoriented surfaces. Kyushu J. Math., 59(2):351-363, 2005, doi: 10.2206/kyushujm.59.351.

[16] A. Kravchenko, B. Feshchenko. Automorphisms of Kronrod-Reeb graphs of Morse functions on 2-torus. Methods Funct. Anal. Topology, 26(1):88-96, 2020, doi: 10.31392/MFAT-npu26_1.2020.07. 
[17] E. Kudryavtseva. Realization of smooth functions on surfaces as height functions. Mat. Sb., 190(3):29-88, 1999, doi: 10.1070/SM1999v190n03ABEH000392.

[18] E. Kudryavtseva. The topology of spaces of Morse functions on surfaces. Math. Notes, 92(1-2):219-236, 2012, doi: 10.1134/S0001434612070243. Translation of Mat. Zametki 92 (2012), no. 2, 241-261.

[19] E. Kudryavtseva. On the homotopy type of spaces of Morse functions on surfaces. Mat. Sb., 204(1):79-118, 2013, doi: 10.1070/SM2013v204n01ABEH004292.

[20] S. Maksymenko. Homotopy types of stabilizers and orbits of Morse functions on surfaces. Ann. Global Anal. Geom., 29(3):241-285, 2006, doi: 10.1007/s10455-005-9012-6.

[21] S. Maksymenko. Homotopy dimension of orbits of Morse functions on surfaces. Travaux Mathematiques, 18:39-44, 2008.

[22] S. Maksymenko. Functions on surfaces and incompressible subsurfaces. Methods Funct. Anal. Topology, 16(2):167-182, 2010, http://mfat.imath.kiev.ua/article/?id=553.

[23] S. Maksymenko. Functions with isolated singularities on surfaces. volume 7, pages 7-66. Proceedings of Institute of Mathematics of NAS of Ukraine, Kyiv, 2010.

[24] S. Maksymenko. Local inverses of shift maps along orbits of flows. Osaka Journal of Mathematics, 48(2):415-455, 2011, http://projecteuclid.org/euclid.ojm/1315318347.

[25] S. Maksymenko. Homotopic types of right stabilizers and orbits of smooth functions functions on surfaces. UMJ, 64(9):1186-1203, 2012, doi: 10.1007/s11253-013-0721-x (in russian).

[26] S. Maksymenko. Deformations of functions on surfaces. Proceedings of Institute of Mathematics of NAS of Ukraine, 17(2):150-199, 2020.

[27] S. Maksymenko. Deformations of functions on surfaces by isotopic to the identity diffeomorphisms. Topology and its Applications, 282(2):107312, 2020, doi: 10.1016/j.topol.2020.107312.

[28] S. Maksymenko, B. Feshchenko. Homotopy properties of spaces of smooth functions on 2-torus. Ukr. Mat. Journ., 66(9):1205-1212, 2014, doi: 10.1007/s11253-015-1014-3.

[29] S. Maksymenko, B. Feshchenko. Functions on 2-torus whose Kronrod-Reeb graph contains a cycle. Methods of Functional Analysis and Topology, 21(1):22-40, 2015, http://mfat.imath.kiev.ua/article/?id $=764$.

[30] S. Maksymenko, B. Feshchenko. Orbits of smooth functions on 2-torus and their homotopy types. Matematychni Studii, 44(1):67-83, 2015, doi: 10.15330/ms.44.1.67-83.

[31] J. D. P. Meldrum. Wreath products of groups and semigroups, volume 74 of Pitman Monographs and Surveys in Pure and Applied Mathematics. Longman, Harlow, 1995.

[32] J. Milnor. Morse theory. Annals of Mathematics Studies, No. 51. Princeton University Press, Princeton, N.J., 1963.

[33] S. P. Novikov. Multivalued functions and functionals. An analogue of the Morse theory. Dokl. Akad. Nauk SSSR, 260(1):31-35, 1981.

[34] S. P. Novikov. The Hamiltonian formalism and a multivalued analogue of Morse theory. Uspekhi Mat. Nauk, 37(5(227)):3-49, 248, 1982.

[35] A. Pajitnov. Circle-valued Morse theory, volume 32 of De Gruyter Studies in Mathematics. Walter de Gruyter \& Co., Berlin, 2006, doi: 10.1515/9783110197976.

[36] A. Prishlyak. Topological equivalence of smooth functions with isolated critical points on a closed surface. Topology Appl., 119(3):257-267, 2002, doi: 10.1016/S01668641(01)00077-3.

[37] A. Ranicki. Finite domination and Novikov rings. Topology, 34(3):619-632, 1995, doi: 10.1016/0040-9383(94)00036-K. 
[38] Georges Reeb. Sur certaines propriétés topologiques des variétés feuilletées. Actualités Sci. Ind., no. 1183. Hermann \& Cie., Paris, 1952. Publ. Inst. Math. Univ. Strasbourg 11, pp. 5-89, 155-156.

[39] F. Sergeraert. Un théorème de fonctions implicites sur certains espaces de Fréchet et quelques applications. Ann. Sci. École Norm. Sup. (4), 5:599-660, 1972, doi: 10.24033 /asens.1239.

[40] V. V. Sharko. Functions on surfaces. I. In Some problems in contemporary mathematics (Russian), volume 25 of Pr. Inst. Mat. Nats. Akad. Nauk Ukr. Mat. Zastos., pages 408 434. Natsīonal. Akad. Nauk Ukraïni, Inst. Mat., Kiev, 1998.

[41] K. Veber, A. Pazhitnov, L. Rudolf. The Morse-Novikov number for knots and links. Algebra i Analiz, 13(3):105-118, 2001.

Received: March 2, 2020, accepted: June 11, 2021.

Bohdan Feshchenko

Topology laboratory, Department of algebra and topology, Institute of Mathematics of National Academy of Science of Ukraine, Tereshchenkivska, 3, KYIV, 01601, UKRAINE

Email: fb@imath.kiev.ua 